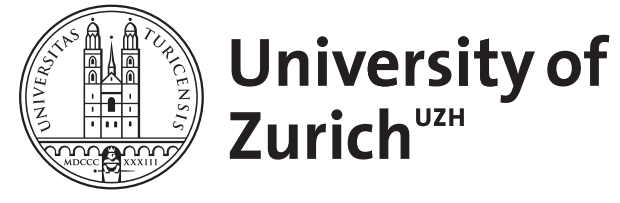

Zurich Open Repository and Archive

University of Zurich

University Library

Strickhofstrasse 39

CH-8057 Zurich

www.zora.uzh.ch

Year: 2002

\title{
Mobiles Customer Relationship Management
}

Hampe, J Felix ; Schwabe, Gerhard

Posted at the Zurich Open Repository and Archive, University of Zurich

ZORA URL: https://doi.org/10.5167/uzh-57161

Book Section

Accepted Version

Originally published at:

Hampe, J Felix; Schwabe, Gerhard (2002). Mobiles Customer Relationship Management. In: Reichwald, R. Mobile Kommunikation. Wiesbaden, Deutschland: Gabler, 0. 


\section{Mobiles Customer Relationship Management}

1. Einführung in das Customer Relationship Management ......................2

2. Mobilität beim Customer Relationship Management........................... 3

3. Systeme und Systemkonzepte für das mobile Customer Relationship

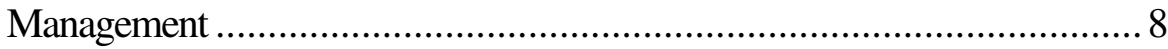

3.1 Aufbau eines CRM-Systems …......................................... 8

3.2 Erweiterungen konventioneller Systeme um mobile CRM-

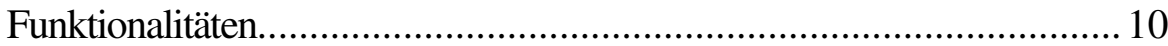

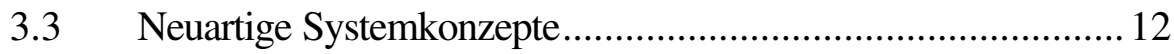

3.4 Die Rufnummer als Schlüssel.......................................... 1817

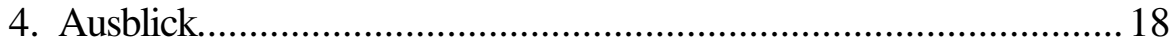

\footnotetext{
${ }^{1}$ Die Autoren erscheinen in alphabetischer Reihenfolge, beide haben zu gleichen Teilen beigetragen.
} 


\section{Einführung in das Customer Relationship Management}

Die ökonomische Attraktivität von Ansätzen zur Kundenbindung - neudeutsch dem Customer Relationship Management (CRM) - lässt sich am einfachsten durch das Konzept der Wechselkosten erläutern: Je höher die Kosten für den Kunden sind, zu einem anderen Anbieter zu wechseln, desto mehr kann ein Unternehmen an ihm verdienen, denn ein ökonomisch denkender Kunde vergleicht immer die Gesamtkosten zweier Angebote. Es muss deshalb im Interesse eines Unternehmens liegen, Wechselkosten bei den Kunden aufzubauen, die für das Unternehmen interessant sind. Daraus leiten sich zwei Kernfragen für ein Unternehmen ab: 1. Wie identifiziere ich die Kunden, die für mein Unternehmen besonders interessant sind und 2. Wie kann es mir gelingen, möglichst hohe Wechselkosten aufzubauen? Antworten auf die erste Frage lassen sich durch Sammeln von Daten und deren Analyse mit Hilfe von statistischen Verfahren gewinnen. Dazu werden sogenannte Data-Warehouse-Ansätze benötigt. Wechselkosten lassen sich dadurch aufbauen, dass ein Anbieter sein spezifisches, kontinuierlich wachsendes Wissen über den Kunden dazu verwendet, ihm ein möglichst weitgehend auf ihn zugeschnittenes Angebot zu unterbreiten. Wechselt der Kunde dann später zu einem anderen Unternehmen, dann muss er einen (zumindest temporären) Verlust an Servicequalität in Kauf nehmen.

Ansätze zum CRM werden mit voranschreitender Digitalisierung des wirtschaftlichen Handelns interessanter, weil auf einen zunehmend umfangreicheren Datenpool z.B. aus ERP-Systemen (z.B. SAP R/3 o.ä.) zurückgegriffen werden kann und weil immer leistungsfähigere Datenverwaltungs- und Analysewerkzeuge zur Verfügung stehen. Hinzu kommt die sich verbreitende Erkenntnis, dass es sehr viel teurer ist, einen neuen Kunden zu gewinnen als einen bestehenden Kunden zu halten (vgl. [Bach/Österle 2000, S. 19] und die dort angegebene Literatur).

Bach und Österle [2000, S. 23 ff] setzen am "Kundenprozess" an und schlagen vor, die CRM-Prozesse Marketing, Verkauf und Service zu unterscheiden, da 
sie unterschiedlich weite Zielgruppen haben: Marketing adressiert den gesamten Markt, der Verkauf identifiziert Interessenten und der Service bestehende Kunden. Zwar sei diese Unterscheidung für analytische Zwecke relevant, bei der Umsetzung ist aber gerade auf eine Integration der Datenbestände und Aktivitäten zu achten. In einem CRM-System werden Kundendaten (z.B. Stamm, Interaktions- sowie Transaktionsdaten), Produktinformationen, Kampagneninformationen und Serviceinformationen zusammengeführt, sinnvolle Aggregate bzw. Cluster gebildet und auf diese dann gezielt zugegriffen. Die wichtigsten Instrumente des CRM sind das Kundenmanagement, das

Kanalmanagement und das Prozess- sowie Wissensmanagement zu seiner Umsetzung im Unternehmen.

Der vorliegende Beitrag zeigt in groben Zügen auf, in welcher Weise CRM um eine Komponente zur mobilen Unterstützung ergänzt werden kann und wie sich CRM durch mobile Unterstützung selbst verändert. Dazu wird in den folgenden Abschnitten zuerst das Thema Mobilität beim Customer Relationship Management diskutiert. Im nachfolgenden dritten Kapitel werden Systeme und Systemkonzepte für das mobile Customer Relationship Management am Beispiel eines konservativen und eines innovativen Szenarios vorgestellt. Ein kurzer Ausblick schließt den Beitrag.

\section{Mobilität beim Customer Relationship Management}

Das Gebiet des Mobile CRM ist - wie das ganze Gebiet Mobile Business gerade erst im Entstehen. Deshalb lässt sich unter diesem Stichwort sehr verschiedenes verstehen. Insbesondere ist zu klären,

- welcher Akteur des CRM mobil ist und

- welche Mobilitätsgrade die Technologie ermöglicht. 
Die Kern-Akteure des CRM sind das Unternehmen (bzw. dessen Vertreter) und die Kunden². Als Mobilitätsgrade lassen sich "stationär" (d.h. an ein Gebäude gebunden wie durch einen stationären PC), "auto-mobil" (d.h. an ein KFZ gebunden wie durch ein Navigationssystem) und "mobil" (d.h. an eine Person gebunden wie durch ein Mobiltelefon oder ein PDA) unterscheiden. Die Einführung einer eigenen Kategorie "auto-mobil" ist gerechtfertigt, da das KFZ ein erhebliche Bedeutung für das persönliche mobile Arbeiten hat (z.B. werden $80 \%$ der Mobiltelefongespräche in den USA vom KFZ aus geführt, so Goldman Sachs 1t. [Newell/Newell Lemon 2001, S. 129]) und weil für das KFZ viele technische Restriktionen nicht gelten, die für Westentaschen-Geräte gelten. So können sie ein größeres Benutzungsinterface und ein höheres Gewicht haben und die Stromversorgung ist deutlich leistungsfähiger.

Sieht man die genannten Mobilitätsgrade für beide Akteure vor, lassen sich insgesamt neun Mobilitätsszenarien des CRM (vgl. Abbildung 1) betrachten.

\begin{tabular}{|c|c|c|c|}
\hline$\underbrace{\text { Mobilitätsgrad }}_{\downarrow \text { Anbieter }} \rightarrow$ & stationär & auto-mobil & mobil \\
\hline stationär & $\begin{array}{l}\text { Unternehmenszen- } \\
\text { triertes CRM }\end{array}$ & $\begin{array}{l}\text { Fahrender } \\
\text { Kunde }\end{array}$ & Mobile Service \\
\hline auto-mobil & $\begin{array}{l}\text { auto-mobile Außen- } \\
\text { dienstmitarbeiter }\end{array}$ & Auto-link & Taxi \\
\hline mobil & $\begin{array}{l}\text { mobile Außen- } \\
\text { dienstmitarbeiter }\end{array}$ & sinnvoll? & Mobile Link \\
\hline
\end{tabular}

\section{Abbildung 1: Mobilitätsszenarien des CRM}

Anmerkung: zuerst werden die Extreme "stationär" und "mobil" vorgestellt und dann begründet, weshalb und inwieweit "auto-mobil" eine besondere Rolle darstellt. Auto-mobil könnte in einer Erweiterung natürlich auch Flugzeug oder

\footnotetext{
2 Ganz generell lässt sich die Diskussion zwar auch auf die Menge der Geschäftspartner sowie die Mitarbeiter eines Unternehmens ausdehnen, von dieser Verallgemeinerung wird aber im folgenden abgesehen.
} 
Bahn umfassen, sobald dort eine entsprechende Technikinfrastruktur bereitgestellt wird.

1. Unternehmenszentriertes CRM: Dieses ist die Hauptausrichtung der meisten CRM-Unternehmenslösungen und für alle anderen Varianten eine unabdingbare Voraussetzung, da aus jedem CRM-Teilprozess wiederum relevante Daten über die Interaktion mit dem Kunden generiert werden. Ziel dieser Basissysteme, auf deren Funktionen und Daten alle anderen Varianten von CRMLösungen zugreifen, ist die nahtlose Abbildung der den Kundenlebenszyklus beschreibenden Merkmalsausprägungen. Da sie keine mobilen Komponenten beinhaltet, wird nicht näher auf ein Szenario aus diesem Bereich eingegangen.

2. Mobile Außendienstmitarbeiter: Der Außendienstmitarbeiter eines Unternehmens sucht den Kunden an dessen Standort für Geschäftsaktivitäten auf, weil es vor Ort einfacher ist, die ungeteilte Aufmerksamkeit des Kunden zu erhalten und auf spezifische Bedürfnisse vor Ort einzugehen. Dort hat der Vertreter Zugriff auf alle relevanten Daten über den Kunden sowie die Produkte des Unternehmens. Hauptvorteile aus Sicht des Unternehmens sind die Aktualität der Daten und die Möglichkeit zur unmittelbaren Rückkopplung mit der Zentrale. Dadurch wird es möglich, ein Geschäft in einem Zug abzuschließen. Dies spart den Beteiligten Zeit und fördert die Tendenz zum Spontanabschluss. Erweiterungen klassischer CRM-Lösungen hin zur Unterstützung von Mobilität zielen zumeist auf dieses Szenario. Technische Hauptherausforderungen sind derzeit die schmalbandigen Datenverbindungen für die Ankopplung zu einem Notebook sowie bei kleineren Geräten (z. B. Mobiltelefon oder PDA) die geringe Größe des Displays. Eine große organisatorische Herausforderung ist der Principal-Agent-Konflikt zwischen Vertreter und Unternehmung bei der Datenerhebung: Es ist ceteris paribus nicht im Interesse des Außendienstmitarbeiters, bisher nur für ihn zugängliche, wertvolle Kontaktdaten in das CRMSystem einzugeben, da diese sein eigenes Kapital sind. Bedrohlich ist nicht nur, dass diese Kundeninformationen damit konkurrierenden anderen Vertretern (des eigenen Unternehmens) zugänglich werden könnten, vielmehr wird ein jeder Außendienstmitarbeiter (subjektiv) leichter austauschbar oder gar ersetzbar. Hier kann ein Unternehmen dadurch seine Interessen besser durchsetzen, 
indem es die Erhebung der notwendigen Kundendaten technisch mit dem Personal Information Management (z.B. Terminkalender) des Vertreters sowie dem unternehmensweiten Kontaktmanagement koppelt. Während der klassische PC nur begrenzt für das Personal Information Management genutzt wird, ist dies ein Haupteinsatzbereich von PDAs und neueren, hochwertigen Mobiltelefonen.

3. Auto-mobile Außendienstmitarbeiter: Der PKW eines Vertriebsmitarbeiters wird zu seinem mobilen Büro ausgebaut. Im Vergleich zu persönlichen mobilen Endgeräten sind in den entsprechenden Geräten im PKW größere Displays und leistungsfähigere Batterien. Dieses mobile Büro dient ihm dann nicht nur zur Unterstïtzung seiner Aktivitäten beim Endkunden, sondern auch der Vor- und Nachbereitung sowie der Abstimmung mit der Zentrale. Onboard oder Offboard-Navigationssysteme weisen den Weg zu Standorten, Suchanfragen bezüglich beim Kundengespräch (etwa auf einer Baustelle) auftretender Materialverfügbarkeits- oder Lieferzeitanfragen sowie Logistikanforderungen werden in Realzeit bearbeitet und somit vollständige Geschäftsvorfälle unmittelbar zum Abschluss gebracht.

4. Mobile Service: Der Kunde hat ein eigenes mobiles Endgerät und die Interaktion wird direkt über dieses Endgerät abgewickelt. Hier sind die "Vorreiter" die Mobilnetzbetreiber. Lokalisationsinformationen und eine weitgehend eindeutige Identifizierbarkeit des Kunden sowie recht starke Authentifikationsmechanismen erlauben die Anpassung der Dienste an den konkreten Kontext und eine Abwicklung einer Transaktion bis zu ihrem Abschluss. In der Literatur (vgl. z.B. [Kenney/Marshall S. 123-124], [Newell/Newell Lemon]) werden als Beispiele orts- und situationsabhängige mobile Werbung genannt ("contextual Marketing" z.B. mit digitalen Coupons mit sehr kurzer Gültigkeit), Notifikation des Personals über Anwesenheit eines guten Kunden (bisher weiß der Supermarkt erst bei der Bezahlung, dass ein guter Kunde mit Kundenkarte eingekauft hat; jetzt weiß man es, sobald er den Laden betritt), situationsabhängige Serviceleistungen (z.B. Benachrichtigung über Änderung im Flugplan für gebuchte Flüge, siehe hierzu weiter unten), Mehrwertdienste (z.B. mobiles Rezeptbuch von Unilever: beim Einkaufen gibt es Ratschläge für Rezepte und ei- 
nen Verweis auf dazu benötigte Produkte); dynamische Preisadjustierungen und Reservierung (z.B. Einreihen in eine virtuelle Warteschlange für eine Attraktion in Disneyland).

Die übrigen Szenarien aus dem Tableau sollen hier nur kurz skizziert werden: Die Idee des Auto-Link ist die Verbindung zweier auto-mobilen Akteure mittels eines ad-hoc-Netzwerkes (z.B. über Bluetooth oder ein Funk-LAN) ${ }^{3}$. Derartige Konfigurationen sind immer dann sinnvoll, wenn die Akteure mobil sind und auf große Displays angewiesen sind (z.B. auf Baustellen, wenn Architekt und Baufirma gemeinsam ad-hoc ein Problem lösen) oder diese Automobile spezielle Aufgaben haben (z.B. Feuerwehr und Polizei). Fahrende Kunden werden derzeit schon von Anbietern von Navigationssystemen mit Verkehrsinformationen versorgt. Kenney und Marshall [S. 125] sehen Autofirmen als neue Intermediäre, die Informationen von fahrenden Kunden (Ort, Tankstand, Fahrtdauer...) an Raststätten oder Hotels verkaufen. Diese bieten den Kunden dann kontextspezifische Dienstleistungen an. In dem "Taxi"-Szenario greift ein mobiler Kunde auf Dienstleistungen eines auto-mobilen Anbieters zurück, beispielsweise wenn er ein Taxi oder eine Pizza bestellt ${ }^{4}$. In der Regel wird die Interaktion durch eine stationäre Zentrale unterstïtzt. Das Szenario "Mobile Link" sieht die Verbindung eines mobilen Anbieters mit einem mobilen Kunden vor. Im einfachsten Fall erfolgt dies über ein Mobilfunktelefongespräch oder das Absetzen einer SMS-Nachricht. Komplexere und innovativere Nutzungsideen reichen in das Gebiet der mobilen Telekooperation [Reichwald et al. 1998], z.B. wenn mittels eines mobilen Endgeräts auch Bilder zu einer anderen Station übertragen werden und über diese dann ein Expertenaustausch (simultan) erfolgen kann (z.B. Notarztunterstützung für Krankenwagen, Spezialistenberatung im Servicefall etc.). Den Autoren ist noch unklar, wie eine sinnvolle Kombination eines mobile Anbieters mit einem auto-mobilen Kunden aussehen kann.

\footnotetext{
${ }^{3}$ Eine interessante Entwicklung für diese Gattung von Anwendungen ist BLIP [Ericsson, 2001], eine Bluetooth basierte Netzinfrastrukturplatform.

4 Tatsächlich kann er heute schon seine Fahrt und die Zusatzdienstleistungen mobil bezahlen [Paybox, 2001].
} 
Schon in der Einleitung wurde darauf hingewiesen, dass die Erhöhung von Wechselkosten CRM für den Anbieter attraktiv macht. Wenn CRM-

Dienstleistungen mit kostenpflichtigen Dienstleistungen verbunden werden können, dann sind diese aus zwei weiteren Gründen für Anbieter attraktiv: Im Vergleich zum Internet sinkt die Markttransparenz (es sei denn es gibt ausgleichende Intermediäre) durch die kontextspezifische Auswahl: Aus einem weltweiten Marktplatz wird wieder ein lokaler Marktplatz mit zeit- und ortsabhängigen Angeboten. Im Vergleich zum konventionellen Marketing mit standardisierten Produkten ermöglichen kontextspezifische und personalisierte Angebote die Aneignung der Konsumentenrente, d.h. die Zahlungsbereitschaft des Kunden wird voll ausgeschöpft. Es ist auch einfacher, Zeitdruck aufzubauen und dadurch die Informationsverarbeitungsmöglichkeit zu reduzieren und Impuls- oder Spontankäufe zu induzieren.

\section{Systeme und Systemkonzepte für das mobile Customer Relationship Management}

Wie kann mobiles CRM umgesetzt werden? Hierauf gehen die nachfolgenden Abschnitte ein. Im Abschnitt 3.1 wird eine Architektur für ein CRM-System vorgestellt und mobile Komponenten in dieses System eingebettet. Die Abschnitte 3.2. und 3.3. diskutieren anhand zweier Szenarien konkrete Herausforderung für die Gestaltung von mobilen CRM-Systemen. Der abschließende Abschnitt 3.4. argumentiert dann, dass die Nutzung von Mobilfunknummern als Kundenschlüssel auch das Potential zur Vereinfachung von konventionellem und mobilem CRM birgt.

\subsection{Aufbau eines CRM-Systems}

Auch ohne ein mobiles CRM-System werden Kunden heute schon mobil bedient, beispielsweise wenn ein Paketdienst die Eingangsbestätigung eines Päckchens auf einem mobilen Endgerät unterschreiben läßt. Traditionell dient hier das mobile Endgerät als direkte Schnittstelle für operative Systeme. Das führt dazu, dass nur die von den operativen Systemen benötigten Kundendaten dau- 
erhaft gespeichert werden - die anderen Kundendaten sind flüchtig. Weiterhin werden die Kundendaten nur für operative Zwecke aufbereitet und ausgewertet. Die Verwendung von Kundendaten für das Relationship-Management erfordert spezielle CRM-Komponenten. Diese CRM-Systeme werden zwischen die Kundenschnittstelle und die operativen Systeme geschaltet (vgl. Abbildung 2Abbildung 2). Zu den im "Back-Office" angesiedelten operativen System gehören ERP-Systeme wie z.B. SAP R/3, Systeme zum Supply Chain Management (SCM), Produktionsplanungs- und Steuerungssysteme (PPS) und weitere ältere "Legacy-Systeme". Die Schnittstellen dieser Systeme zueinander und für externe werden im Rahmen einer Enterprise Application Integration (EAI) integriert. Auf diese einheitliche Schnittstelle greifen auch die eigentlichen CRMSysteme zu. Man unterscheidet hier zwischen "Operational CRM" für die eigentliche Arbeit an der Kundenschnittstelle, "Content Management" für die Verwaltung von Dokumenten und Wissen über Kundenkontakte sowie "Analytical CRM" zur statistischen Auswertung von quantitativen Daten aus operativen Systemen, z.B. um Kunden gemäß ihres Bestellverhaltens zu segmentieren. 


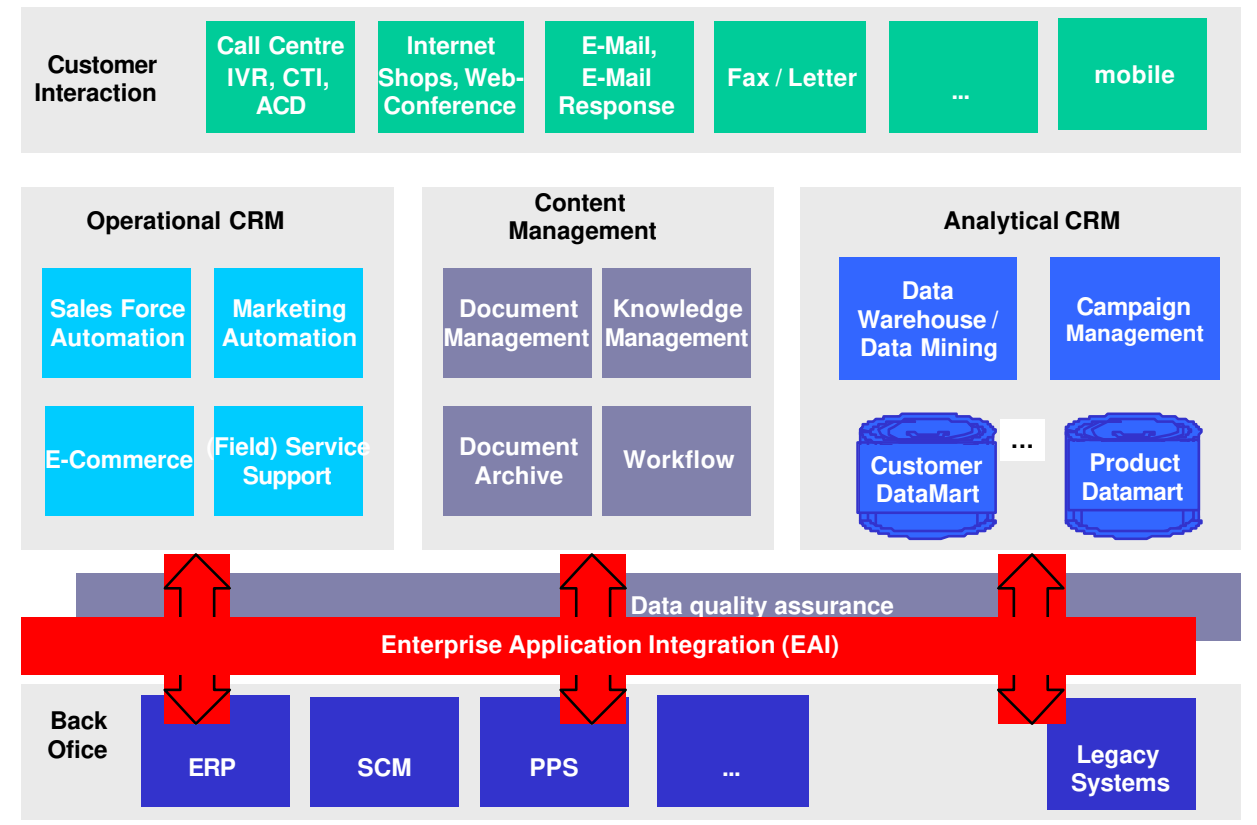

\section{Abbildung 2: Architektur eines CRM-Systems}

Die eigentliche Interaktion mit dem Kunden kann über verschiedene Kanäle stattfinden: Der Kunde kann beispielsweise über ein Call Center (vgl. [Hampe/Schönert, 1997]), einen Außendienstrepräsentanten vor Ort (dies schließt den Filialmitarbeiter ein), das Web, E-Mail, Fax oder konventionelle Briefaktionen kontaktiert werden oder seine Wünsche an das Unternehmen herantragen. äußern und vom Unternehmen bedient werden.

\subsection{Erweiterungen konventioneller Systeme um mobile CRM- Funktionalitäten}

Zu welchen Änderungen führt eine Erweiterung der obigen CRM-Architektur, wenn mobile Komponenten hinzugefügt werden? Dies sei am Beispiel eines mobilen Versicherungsvertreters beschrieben: 
Szenario 1: Eine Versicherung stattet ihre Repräsentanten mit einem mobilen Computer (z.B. einem Notebook) aus, der über Mobilfunk mit der Zentrale verbunden ist. Vertreter und Kunde greifen vor Ort nicht nur auf die aktuellen Konditionen zurück, sondern sie können dort auch das Geschäft weitgehend zu einem Abschluss bringen, da die Daten (Bonität, Schadenshistorie, Risikoeinstufung) in der Zentrale online geprüft werden können. Der Zugriff auf die Kundendaten eröffnet weiterhin die Möglichkeit dazu, dem Kunden gezielt weitere Produkte anzubieten (cross- und up-selling). Diese Bemühungen basieren dann nicht nur auf der inhaltlichen Nähe zweier Produkte (z.B. Autohaftpflicht- und Insassenschutzversicherung), sondern auch auf dem bisherigen Versicherungsbestand des Kunden, dem Wert des Kunden für das Unternehmen und den von ihm gespeicherten Präferenzen.

In diesem Szenario stellt der mobile Datenzugriff einen zusätzlichen Kanal (vgl. Abbildung 2Abbildung 2 oben rechts) für die Interaktion mit dem Kunden dar. Operative Systeme und CRM-Systeme bleiben funktional weitgehend unverändert; zusätzlichen Aufwand generiert nur die mobile Anbindung. Eine komplizierte Herausforderung stellt bei diesen Multi-Kanal-Architekturen die Koordinierung von Informationsströmen in zeitlicher, inhaltlicher sowie rechtlicher Hinsicht dar. Die meisten derzeit in der Presse und in Produktankündigungen vorgestellten Lösungen zum mobile CRM folgen dieser Idee eines zusätzlichen mobilen Kanals in einer Multi-Kanal-Architektur. Wegen der tatsächlich noch immer existierenden Systemrestriktionen (Bandbreite, Robustheit, Verbindungskosten etc.) werden dabei vielfach auch semi-mobile Lösungskonzepte verfolgt, bei denen zunächst Daten in einem offline-Speicher gehalten werden, die erst zu einem späteren Zeitpunkt und bei Verfügbarkeit einer geeigneten Netzanbindung auf zentrale Systeme überspielt werden.

In einigen Branchen sind derartige Ansätze zur mobilen Unterstützung der Dienstleistungsprozesse beim Kunden schon seit längerem verbreitet, zum Teil mit qualitativ hochentwickelten Lösungen. Zu nennen sind hier exemplarisch der Außendienst im Finanzdienstleistungsbereich, die KfZ-Schadenschätzung oder die kombinierte Bestands- und Bestellerfassung im Handel. Die Einsatzspanne 
im letztgenannten Anwendungsfeld kann von der DECT-basierten Bestellerfassung im Gartenlokal bis zur Disposition in Großlagern über WLAN-Technik reichen. In vielen Fällen handelt es sich um Unternehmenslösungen, weniger um Massendienste für den Endkundenbereich mit direkter Interaktion von Kunden.

Diese Gattung von Kundenunterstützungssystemen werden typischerweise von einem Unternehmen oder einem von ihm beauftragten Dienstleister betrieben, die Arbeitsleistung der Bedienung wird also nur in einigen Varianten auf die Kundenseite übertragen. Aus wirtschaftlicher Sicht für den Dienstleister attraktiver sind aber gerade jene Lösungen, bei denen dem Kunden eine für ihn personalisierte Arbeitsumgebung zur Verfügung gestellt wird, mit der er seine Aufgaben selbst erledigt. Bekannte Beispiele hierfür sind das electronic-Banking, die web-EDI Ansätze zum eProcurement (z.B. für Bürobedarf) oder auch die schon klassischen Großhandelsbestellsysteme im Pharmabereich. Diese Systemkonzepte haben wenig Neuartigkeit, sie bieten aber unter Rationalisierungsaspekten erhebliche Potentiale, wie sich an dem sich vorrangig im Bankbereich herausbildenden spezifischen Preisgefüge (z.B. Gebührengutschrift bei Tätigung von online-Überweisungen etc.) ableiten läßt.

Neben der Filiallösung sowie dem Call Center entwickelt sich das Web zum favorisierten Kundeninteraktionkanal. Es scheint absehbar, dass für einfache Finanztransaktionen mobile Lösungen eine mindestens gleiche Bedeutung erlangen [Durlacher, 1999; Natsuno, 1999].

\subsection{Neuartige Systemkonzepte}

Erweiterungen traditioneller CRM-Systeme um mobile Datenzugriff schöpfen die Potentiale der Mobilkommunikation bei weitem noch nicht aus. Das folgende Beispiel zeigt, wie die Ubiquität, hohe Erreichbarkeit und Sicherheit in Verbindung mit Lokalisierungsinformationen, sofortiger Verbindung ("always-on") und Personalisierung neuartige Dienste für den Kunden erlauben, die weit über das Modell eines zusätzlichen Kanals hinausgehen. CRM-Systeme und mobile Mehrwertdienste werden dabei funktional untrennbar miteinander verwoben. 
Szenario 2: Scenario für mobile CRM unter Einsatz von Location Based Services:

Die Fluggesellschaft Airline bietet als Teil ihres Customer Relationship Managements folgenden Anreisedienst für ihre hoch profitablen Kunden (,,SenatorKlasse") an: Kunde Müller hat seinen Abflug von Frankfurt um 18:30 Uhr nach New York bestätigt. Es wird festgestellt, dass er zum genannten Kundensegment gehört, für ihn werden deshalb spezifische Serviceangebote freigeschaltet sowie die Option zur Personalisierung dieser Dienste geboten. Neben Präferenzen bezüglich der Sitzplatz- oder Speisewahl umfasst dies auch die Anforderung von Unterstützungsleistungen vor und nach dem Flug. Am Tag seines Abflugs erhält er deshalb einen Anruf auf seinem Mobiltelefon (voreingestellt), bei dem ihm angeboten wird, ihn auf seinem Weg zum Flughafen zu unterstïtzen. Dies umfasst die Benachrichtigung über eventuelle Änderungen in seinem Flugplan oder auch Verkehrsplanungsinformationen für den Weg zum Flughafen und vor Ort. Sobald der Kunde diese Serviceangebot explizit (z.B. durch Eingabe eines Freischaltungscode) akzeptiert, verbindet sich die Fluggesellschaft mit dem Navigationssystem im Auto des Kunden (oder mit seinem Mobilfunkgerät). Unter Rückgriff auf verfügbare aktuelle Verkehrsinformationen, den Aufenthaltsort (location based Service) und die mittlere Reisgeschwindigkeit des Kunden beobachtet ein Leitsystem der Fluggesellschaft den Reiseverlauf des Kunden. Im Normalfall (d.h. bei erwarteter rechtzeitiger Ankunft) beschränkt sich die Fluggesellschaft darauf, ihm eine Parkplatzreservierung anzubieten, einen priorisierten Check-in anzubieten sowie das Abflug-Gate mitzuteilen. Sollte aber absehbar sein, dass die Zeit knapp wird, werden zusätzliche Dienstleistungen zur Beschleunigung der Abfertigung in die Wege geleitet: Die Fluggesellschaft übernimmt das Parken des Autos, sorgt für eine beschleunigte Gepäckabfertigung, leitet den Kunden auf direktem Weg zum Gate und benachrichtigt bei Bedarf auch das wartende Flugzeug. Zuvor kann zusätzlich der Navigationsservice der Fluggesellschaft Alternativen zur Anfahrt zum Flughafen ermitteln und vorschlagen, z.B. durch Umfahrung eines Staus oder den Wechsel des Verkehrsmittels. 
Sollte die Verspätung so groß werden, dass der Kunde sein Flugzeug nicht mehr erreichen kann, dann wird ihm noch vor Erreichen des Flughafens eine Umbuchung auf ein anderes Flugzeug oder ein anderes Verkehrsmittel (bei Inlandsflügen) angeboten. Bei diesen meist bereits stresserzeugenden Situationen bzw. Aktionen wird die Kommunikation über speziell geschulte Mitarbeiter in Communication Centern geführt, d.h. der Sprachkanal genutzt. Sollte sich hingegen der Abflug verzögern, dann kann der Kunde ggf. noch vor seiner Abfahrt zum Flughafen, sonst während der Hinfahrt benachrichtigt werden, dass er sich Zeit lassen kann. In jedem Fall wird nach Erreichen des Flughafens der Kunde davon unterrichtet, dass der Lokalisierungsdienst abgeschaltet wird.

Für den Kunden bedeutet dieses Dienstportfolio, dass er für eine begrenzte Zeit private Daten für die Bequemlichkeit einer betreuten Anreise preisgibt. Dies setzt zum einen die Leistungsfähigkeit des Dienstes und zum anderen ein Vertrauen in die Verlässlichkeit des Dienstanbieters beim Umgang mit sensiblen Daten voraus. Es ist offensichtlich, dass ein solcher Anreisedienst nur unter Rückgriff auf ein CRM-System möglich ist, dass dieses aber alleine nicht ausreicht. Das CRM-System wird zur Selektion der in Frage kommenden (Senator-) Kunden und zur Ermittlung und Verwaltung von deren Präferenzen (wurden und werden solche Dienstangebote gewünscht oder der fühlt er sich eher gestört) benötigt. Für Informationen zu Flügen und Buchungen greift ein CRMSubsystem auf andere operative Systeme zurück. Hinzu kommt der Zugriff auf spezifische mobile Mehrwertdienste. In dem beschriebenen Szenario sind dies Ortsinformationen über den Kunden, z.B. vom Mobilfunkbetreiber, Routenund Verkehrsinformationen (von Navigationsdiensten), Parkhausreservierungssysteme oder auch mobile Bezahlsysteme (z.B. bei einem Wechsel des Verkehrsmittels bei der Anreise). Konventionelle, geschlossene CRM-Systeme sind somit um geeignete Schnittstellen zur Anbindung solcher Systeme zu ergänzen. Zudem müssen die Datenmodelle so vom Entwurf her erweitert werden, zusätzliche Fremdinformationen für die Personalisierung von Angeboten aufzunehmen und für das Contolling oder auch das analytische CRM zu verdichten. Die Gestaltung dieses Airline-Beispiels wirft weitere Fragen auf, die bei innovativen mobilen CRM-Lösungen in den Vordergrund rücken: 
1. Stickiness des Kunden: Die Fluggesellschaft steht dann vor einem Dilemma, wenn es im Kundeninteresse steht, ihn an einen Konkurrenten weiterzuleiten, z.B. wenn ein verspäteter Kunde seinen gebuchten Flug nicht mehr erreichen kann und eine Konkurrenzmaschine früher zum geplanten Ziel fliegt als eine eigene. Hier ist abzuwägen zwischen der Möglichkeit, den Kunden zu verärgern, weil dieser zum einen lange warten muss und zum anderen erfährt, dass es eine Alternative geben würde. Andererseits besteht die Gefahr, dass der Kunde abwandert, weil er bei dieser Gelegenheit eine andere Fluggesellschaft schätzen lernt. Die Stärke der Bindung eines Kunden an ein Unternehmen bezeichnet

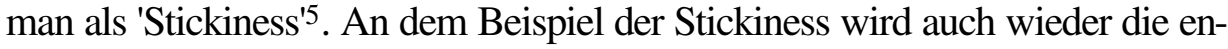
ge Verkoppelung von traditionellem CRM-System, operativen Systemen und den neuen mobilen Diensten deutlich: Daten zur Stickiness eines Kunden (also zu seiner Treueneigung) lassen sich aus dem analytischen CRM extrahieren ${ }^{6}$, welches sich wiederum aus den bisherigen Buchungsdaten, den gespeicherten Daten über die bisherige Inanspruchnahme des Anreisedienstes und anderer Serviceangebote speist. Weiterhin werden direkt Daten aus der laufenden Inanspruchnahme des Anreisedienstes (z.B. darüber, wer für das Verpassen des Flugs verantwortlich ist) benötigt. Die Konsequenzen dieser Analyse fließen dann direkt in die operativen Systeme zur Unterstützung der Agenten im Communication Center ein.

2. Gestaltung des Dialogs: Gerade weil Kunden jederzeit und überall erreichbar sind, muss der Dialog mit ihnen mit Sorgfalt gestaltet werden. Oberster Grundsatz sollte sein, dass mit Kunden nur dann ein Dialog begonnen wird, wenn sie im Prinzip die Erlaubnis dazu gegeben haben (Permission Based Marketing [Schwarz, 2001]). Ist diese Erlaubnis nur sehr allgemein gewesen, dann sollte zu Beginn einer Interaktion wiederum die konkrete Erlaubnis des Kunden abgefragt werden. Eine wesentliche Herausforderung ist es hierbei, herauszufinden, wann ein geeigneter Zeitpunkt dazu ist und an welches Ereignis am geschicktesten angeknüpft wird. Im Airline-Beispiel könnte als Anknüpfungspunkt für das konkrete Dienstangebot die Ticketausstellung gewählt werden; der ge-

\footnotetext{
5 vgl. [Walczuch et al. 2001]; der Begriff ist eng verwandt mit "Lock-In" [Shapiro/Varian 1999].

6 i.d.R. jedoch nicht für die Einzelperson, sondern für eine Gruppe
} 
eignete Zeitpunkt zur Serviceaktivierung ist zeitnah zur voraussichtlichen Abfahrt oder durch Initiierung des Kunden selbst. In Abhängigkeit von der Wichtigkeit und der Dringlichkeit der Interaktion ist das Medium zu wählen. Während das Angebot, den Wagen parken zu lassen, noch über eine SMS-

Nachricht abgewickelt werden kann, eignet sich für sensible, interaktionsintensive, nicht deterministische Probleme (wie z.B. die Umbuchung eines Flugs) eher ein Telefonanruf aus einem Call Center.

Weiterhin sind die bereits angesprochenen Grenzen mobiler Endgeräte zu beachten: stark limitiertes Interface, schwierige Bedienung und häufig nicht leistungsfähig bei der Datenkommunikation. Für die Sprachkommunikation sind diese Geräte jedoch unübertroffen und erlauben zudem eine sehr persönliche, situative und iterative Interaktion. Chancen für mobile Kundenservices liegen deshalb in der situationsabhängigen sinnvollen Kopplung von Information und Kommunikation sowie abschließenden gesicherten, aber kurzen Transaktionen. Chancen liegen weiterhin in der Gestaltung eines Medienmix, insbesondere Links zwischen papiergestiutzten Informationen (zum Erheischen von Aufmerksamkeit), Informationen auf einem mobilen Geräte (zur kontextabhängigen Notifikation), mündliche Kommunikationsmöglichkeiten über Handy (für Rückfragen) und webbasierten Informationen (z.B. zum Aufbau von personalisierten Portals oder Communities). Die große Herausforderung ist dabei die Abstimmung der Kanäle aufeinander (Multichannel-Integration).

3. Privatsphäre und Bequemlichkeit (convenience): Die geringe Größe mobiler Benutzungsschnittstellen und die Anforderung leichtester Bedienbarkeit durch die Nutzer führen dazu, dass mobile Dienste vielfach auf PushInformationen abstellen werden. Dies kann beim Kunden zum Gefühl eines Kontrollverlustes und der Bevormundung führen. Newell und Newell Lemon [S. 162] schlagen deshalb abgestufte Filter für die Erreichbarkeit vor: Je nach Wichtigkeit und Dringlichkeit (für den Kunden!) werden die Nachrichten in verschiedenen Bereichen abgelegt und der Dialog unterschiedlich gestaltet. Während die Nachricht, dass ein geplanter Flug ausfällt, sofort und über einen Telefonanruf aus dem Call Center mitgeteilt wird, kann ein Sonderangebot zur Nutzung von Frequent Flyer Miles über die normale Post versendet werden 
und das Angebot, den Wagen am Flughafen zu parken über eine SMSNachricht. Wesentlich ist hierbei, dass der Anbieter zwar Vorschläge über die Einstellung der Filter machen kann, aber die Kontrolle über die Filter immer dem Kunden obliegt. Dies bedeutet allgemein, dass die Initiative zu einer personalisierten Geschäftstransaktion sich vielfach vom Lieferanten zum Kunden verlagern sollte, da er zumindest den Initiierungsschritt tun muss, indem er signalisiert, dass er für einen Dienst empfänglich ist.

Ein noch offenes Problem besteht darin, wie eine missbräuchliche Nutzung oder Weitergabe der erfassten Daten verhindert werden kann. Schon die Segmentierung der Kunden aufgrund erfasster Transaktionsdaten ist nicht im Interesse aller Kunden (insbesondere nicht derer, die dadurch in eine niedrige Serviceklasse gelangen). Auch eine Weitergabe der Daten an andere Unternehmen ist schwer zu verhindern. Letztendlich ist das Problem aus Sicht des Kunden nur dadurch zu lösen, dass seine Daten nicht direkt bei den Dienstleistern gespeichert werden, sondern bei darauf spezialisierten vertrauenswürdigen Intermediären. Dies liegt aber nicht im Interesse der Unternehmen; bewusste Kunden müssen sich deshalb schon heute vielfach entscheiden, wie viel ihnen die Bequemlichkeit an Verlust der Privatsphäre wert ist.

Trotz des Aufwands können sich diese neuen Systemkonzepte für den Anbieter ökonomisch lohnen: Während die Mobilitätsunterstützung in Szenario 1 (Versicherungsvertreter vor Ort) die Wechselkosten für den Kunden nicht erhöht, kann das Szenario 2 (Fluggesellschaft Airline) zu deutlich höheren Wechselkosten führen. Wechselkosten entstehen zu Beginn durch den wahrgenommenen Nutzen des Kunden. Je besser die Fluggesellschaft sich auf den Kunden einstellt, das heisst auch: je mehr Daten sie über seine Präferenzen gesammelt hat, desto höher werden die Wechselkosten für den Kunden. Selbst wenn eine andere Fluggesellschaft ihm einen vergleichbaren Dienst anbietet, muss diese erst die Präferenzen des Kunden erlernen (das heisst insbesondere: ausreichende Daten sammeln). 


\subsection{Die Rufnummer als Schlüssel}

Mobile Technologie bietet nicht nur die Chance zu immer besseren, aber auch immer komplexeren Dienstleistungen, sondern kann auch zur Vereinfachung bestehender und innovativer CRM-Lösungen führen: Ein modernes GSMTelefon vereinigt die Funktionen eines Kommunikationsgeräts, eines Informationsgeräts, einer Scheckkarte, eines Ausweis sowie einer Kundenkarte. Der wesentliche Unterschied zu internetbasierten Systemen besteht in der eindeutigen Identifizierbarkeit des Endgerätes über die SIM-Karte und damit indirekt des Vertragskunden. Es gibt eine relativ hohe Systemsicherheit bei GSMbasierter Mobilkommunikation, bei angeschalteter Rufnummernübermittlung ist die Identifikation in idealer Weise nutzbar (z.B. als Kundennummer bei Autovermietungen, zur Anrufervorqualifizierung und daraus abgeleiteter Serviceprozeduren). Es liegt deshalb nahe, den Kunden auch systemintern über seine Mobiltelefonnummer zu identifizieren, d.h. die Mobiltelefonnummer als einheitlichen Primärschlüssel für die operativen Systeme und das CRM-System zu verwenden. Dies impliziert eine besondere Bedeutung der Rufnummernportabilität, um dieses personengebundene Merkmal möglichst lange an den Kunden zu koppeln. Dies ist in beidseitigem Interesse (es ist tatsächlich eine Herausforderung an das churn-management ${ }^{7}$ der Mobilfunkprovider, einer Spezialdisziplin im CRM), tatsächlich steht die Umsetzung dieses Vorhabens aber noch aus.

\section{Ausblick}

Mit dem Aufkommen der Diskussion um die technologischen Merkmale der Mobilfunkkommunikation der 3. Generation wurde anfangs die Notwendigkeit qualifizierter, ertragssichernder Mehrwertdienste für diese Netzinfrastrukturen nicht stark genug diskutiert. Inzwischen hat sich dies gewandelt, da für die Basisdienste nur wachstumslimitierte Ertragsströme prognostiziert werden [TIMElabs 2000]. Bei der Erforschung neuer Mehrwertdienstkonzeptionen wird es -ähnlich wie im Airline-Beispiel- darauf ankommen, spezifische Kunden-

\footnotetext{
7 Churn bezeichnet im Englischen den Wechsel eines Kunden zur Konkurrenz . Churn Management versucht dies insbesondere für profitable Kunden zu verhindern, indem es Wechselwilligkeit rechtzeitig identifiziert und darauf reagiert; vgl. auch [Lowenstein 2002].
} 
segmente mit maßgefertigten Serviceprodukten zu konfrontieren. Die Kenntnis solcher Kundengruppen mit hinreichendem Nachfragepotential sowie abschätzbarer Bereitschaft zur direkten oder indirekten Vergütung der Mehrwertdienstnutzung wird auf der Basis der aus CRM-Studien gewonnen Erkenntnisse aufsetzen. Gleichzeitig werden diese Systeme dann auch für den Betrieb, die Tariffierung sowie die Weiterentwicklung der Dienstkonzepte nach Kundenanforderungen heranzuziehen sein. Einhergehend mit der massiven Durchdringung aller Gruppen und Lebensbereiche mit den Varianten der Mobilkommunikation wird daher gerade dem mobile CRM eine zunehmende Bedeutung seitens der Autoren vorhergesagt.

\section{Literatur}

BACH, VOLKER/ ÖSTERLE, HuBERT: Customer Relationship Management in der Praxis, Heidelberg et al. 2000.

DURLACHER RESEARCH. Mobile Commerce Report. http://www.durlacher.com/research/res-reports.asp ; 1999 [Version: 6.2.2001]

ERICSSON CORP.: Bluetooth Local Information Point. http://www.ericsson.com/blip/ [Version 22.10.2001]

HAMPE, J. FELIX / SCHÖNERT, SILKE : Call Center. In: Wirtschaftsinformatik, 39, 2/1997, S. 173-176.

KENNY, DAVID/ MARSHALL, JOHN: Contextual Marketing. Harvard Business Review November-Dezember 2000, S. 119 - 125.

LOWENSTEIN, MICHAEL: Churn Models and Customer Reincarnation, http://www.crm-forum.com/library/art/art-125/art-125.html [Version vom 8.3.2002].

NATSUNO, TAKESHI: "i-mode" Toward Mobile Multimedia in 3G. NTT Mobile Communications Network, Inc.(NTT DoCoMo); Votragsunterlagen, Oct. 1999 
NeWEL, FREDERICK/ NeWELl LEMON, KATHERINE: Wireless Rules - New Marketing Strategies for Customer Relationship Management Anytime Anywhere, New York et al. 2001.

PAYBOX AG: http://www.paybox.de/ [Version 22.10.2001]

PREIBNER, ANDREAS: Marketing im E-Business, München et al. 2001.

REICHWALD, RALPH/ MÖSLEIN, KATHRIN/ SACHENBACHER, HANS/

ENGLBERGER, HERMANN/ OLDENBURG, STEPHAN: Telekooperation -

Verteilte Arbeits- und Organisationsformen. Springer, Heidelberg u.a. 1998.

SCHWARZ, THORSTEN: Permission Marketing. 2. Aufl.; Max Schimmel Verlag, Stuttgart, 2001.

SHAPIRO, CARL/ VARIAN, HAL: Information Rules, Harvard Business School Press, Cambridge 1999.

TIMELABS RESEARCH CENTER. Winning in Mobile eMarkets. Diebold

Deutschland GmbH, Eschborn, 2000.

WALCZUCH, RITA/ VERKUIJLEN, MARCEL/ GEUS, BAS/ RONNEN, URSELA:

Stickiness of commercial virtual communities; Merit Infonomics Research Memorandum Series Nr. 22-2001, Maastrich Economic Research Institute on Innovation and Technology, Maastrich 2001,

http://meritbbs.unimaas.nl/rmpdf/2001/rm2001-022.pdf [Abruf 8.3.2002] 